\title{
Conduct of Neuroanesthesiology and Neurocritical Care Final Examination during COVID-19: Challenges and Experience!
}

\author{
Sourav Burman' Gyaninder P. Singh1,॰ Keshav Go
${ }^{1}$ Department of Neuroanaesthesiology and Critical Care, All India
Institute of Medical Sciences, New Delhi, India
}

J Neuroanaesthesiol Crit Care:2020;7:160-162

\section{Introduction}

The pandemic of coronavirus disease 2019 (COVID-19) has significantly disrupted the medical education. ${ }^{1}$ It has posed unique practical and logistic challenges pertaining to both medical education and examination process for accreditation. $^{2}$ This pandemic has not only compelled the medical educators to think how to provide continuity of teaching and certification assessments for trainees during the present times but may forever change the way how future physicians are educated. ${ }^{1,3}$ The novel challenge posed by the present scenario has prompted institutions across the world to move to web-based virtual platforms for medical teaching, conferences and multidisciplinary meets, and even for conduct of medical examinations. ${ }^{1,4}$

Conduct of examination involves meticulous planning of the entire process and appropriate measures to uphold the sanctity of the examination process. University examinations in India for medical courses are held in two parts: theory (written examination) and clinical (viva-voce). Conduct of these examinations in a fair and most appropriate manner requires extensive preparation and execution. This is even more challenging during the current COVID-19 pandemic, as there is a pressing need to avoid contracting the disease and to ensure adequate safety and protection of the examiners as well as examinees at all times. Although the written examination can be conducted in a large ventilated space with maintenance of social distancing, hand hygiene, and mask etiquettes, the conduct of clinical practical examination is more onerous as it requires interaction between the examinee, patients, and the examiners. We share our experience on successfully conducting two virtual practical-clinical
Address for correspondence Gyaninder P. Singh, MD, DM, Department of Neuroanaesthesiology and Critical Care, All India Institute of Medical Sciences, Neurosciences Centre, Room No. 711 (7th Floor), New Delhi 110029, India

(e-mail: drsingh_gp@yahoo.co.in).

examinations (DM [Neuroanesthesiology and Critical Care], and Fellowship [Neurocritical Care] certification), in a safe manner during the present pandemic. We formulated an indigenous approach that was never used before for conduct of any examination in our department.

\section{Conduct of Examination}

The exercise to conduct the virtual practical-clinical examinations began with the prior planning that involved identifying the specific requirements and managing the logistics, anticipating the possible problems and how to overcome them, and forming strict protocol to reinforce meticulous social distancing, robust mask etiquettes, and frequent hand hygiene. Prevailing travel restrictions in the wake of countrywide lockdown made it difficult for the external examiners (especially from out of the city) to be present in person during the examination. Thus, an online video-cum-audio interface was set up in a well-ventilated and spacious room for virtual conduct of examination. The application for online virtual meetings (Microsoft Teams software) was installed in the computer in the examination room, and by the external examiners in their respective devices, to connect virtually during the examination. Two trial runs were performed before the day of examination in which all the external and internal examiners participated to ensure the smooth functioning of the system. A high Internet streaming speed is an important factor in ensuring seamless and hassle-free video-conferencing. ${ }^{5}$

Another challenge during the present time was to curtail physical proximity between the candidate and the patient for history taking and clinical examination of the patients.
Dol https://doi.org/ $10.1055 / \mathrm{s}-0040-1717832$ ISSN 2348-0548.
(C) 2020. Indian Society of Neuroanaesthesiology and Critical Care.

This is an open access article published by Thieme under the terms of the Creative Commons Attribution-NonDerivative-NonCommercial-License, permitting copying and reproduction so long as the original work is given appropriate credit. Contents may not be used for commercial purposes, or adapted, remixed, transformed or built upon. (https://creativecommons.org/licenses/by-nc-nd/4.0/). Thieme Medical and Scientific Publishers Pvt. Ltd., A-12, 2nd Floor, Sector 2, Noida-201301 UP, India 
To overcome this, a list of 12 cases ( 6 for long format and 6 for short format) was prepared in consultation with external examiners and details of history, examination findings, investigation, and diagnosis were taken from medical records of the patients. The case summaries were prepared in a standard format in digital form. Two sets of summaries were prepared: one version for the students that contained only the history and examination findings and the other version for the examiners that contained additional investigations and final diagnosis. All investigations pertaining to the individual cases including laboratory reports, computed tomography (CT) and magnetic resonance imaging (MRI) were collected in digital format and stored in a dedicated folder in the computer prior to examination that could be simultaneously shared on-screen with the candidate in examination room and examiners at remote locations during the examination. Moreover, the ergonomics of seating was such planned that adequate distance is maintained between the people in the room and the virtual examiner gets a holistic view of the entire examination room, with a focused eye on the candidate ( - Fig. 1A, B). No person other than the candidate, the internal examiners, and faculty coordinating the exam were allowed inside the examination room.

On the day of examination, each candidate was randomly allotted three cases (one long format and two short format) in the presence of two internal examiners and one external examiner (from the same city) present physically and another external examiner (from different city) who joined online. The student version of case summaries was provided to the candidates initially (comprising brief history of presenting complaints and examination findings). A different set of case summaries having complete details of patients including investigations and diagnosis was provided to the examiners. The candidates were called upon sequentially to present their case in front of internal examiners present in person and the external examiners connected through video-conferencing. Adequate social distancing, mask etiquette, and hand hygiene were reinforced periodically. ${ }^{5}$ All relevant clinical information was not shared with the candidate and some were intentionally withheld to evaluate the candidate if he could elicit such information. A set of questions was asked such as what additional symptoms or history the candidate will like to elicit or possible differential diagnosis after the history or what investigations he would like to have for the diagnosis and before planning for anesthetic management and so on. When required the relevant information about the patient's investigations and imaging were shared on the screens of all examiners and candidate simultaneously for discussion. The examiners were free to interrupt and generate related discussions to gauge the candidate's depth of knowledge.

For viva-voice, the external examiners screen-shared the contents from their computers such as images of electrocardiograms, arterial blood gases reports, various graphs and traces, X-ray, CT, MRI, and neurointervention images as PowerPoint presentation and interviewed the candidates. Besides this, the complete collection of all relevant drugs, devices, and equipment was kept in the examination room from which the candidates were asked to pick randomly and answer the questions. For evaluation of theory papers, the answer sheets were converted into digital format and transmitted to the external examiners over a secure platform by the examination section of the institute. ${ }^{5}$ Thus, the candidates were evaluated in all aspects (as during the examination held in the previous years) and at the end of the examination, all the examiners discussed the performance of each candidates, prepared the results, and signed it (digitally by virtual external examiner).

\section{Our Experience and Learning}

Some salient points that were a learning experience for us are as follows: (1) It is important to anticipate, plan, and prepare well in advance. Multiple trial runs may be required to know and overcome the shortcomings before the conduct of actual examination; (2) Access to high-speed Internet facility at both ends is extremely important to allow live streaming seamlessly; (3) The examination room must be well illuminated and background noise must be curtailed to minimum; (4) The
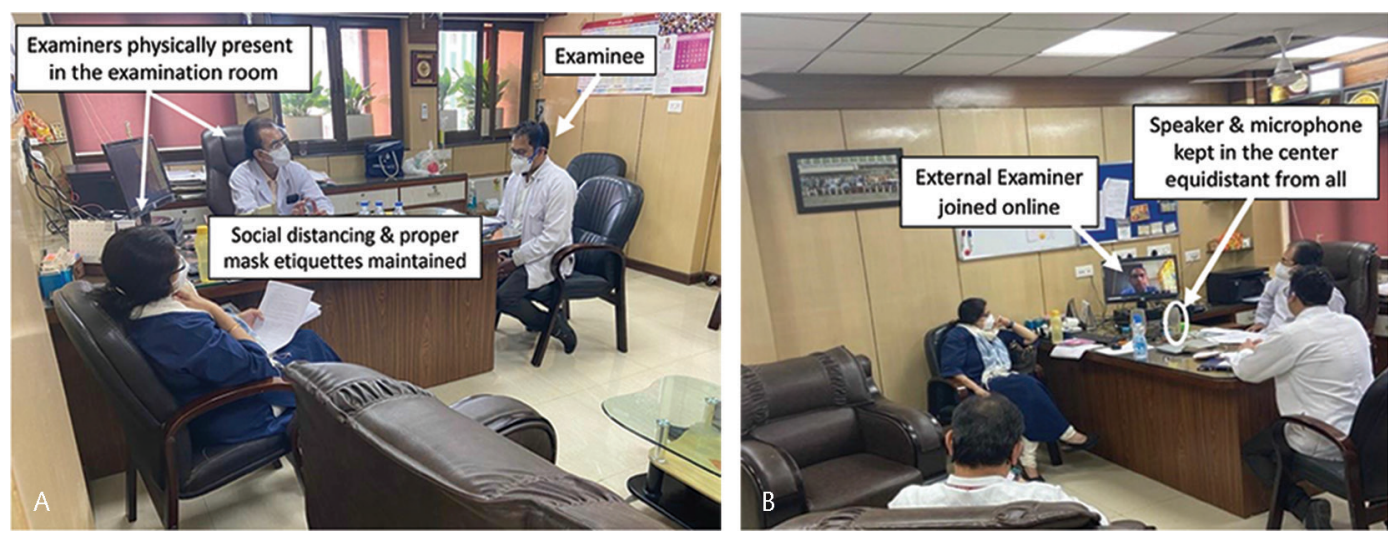

Fig. 1 Seating ergonomics in the examination room (A), adequate social distancing, and proper mask etiquettes followed during the examination. (B) The computer screen is clearly visible to the candidate and the examiners in the examination room and the virtual (online) examiners get a holistic view of the entire examination room, with a focused eye on the candidate. 
web camera should be at an elevated position for a holistic view of all the members in the room; (5) The single speaker-cum-microphone should be placed on the center of the table equidistant from the internal examiners and candidate to capture the voice of all clearly. Multiple speakers in the close vicinity cause audio distortion. Mobile phones and other nonessential electronic devices that can potentially cause audio interference should be removed; (6) The computer screen should be at a comfortable height and should be clearly visible to the candidate as well as the examiners present in the room. The emails or other notification prompts on screen as well as system updates should be disabled for uninterrupted two-way communication during the examination; (7) There should be least minimum traffic in the room to avoid background disturbances; (8) Proper disinfection of examination room, and all exam-related material like drugs, instruments, scans, files, and folder must take place before and after handling; (8) Frequent hand hygiene, proper mask etiquettes, and social distancing should be strictly adhered to at all times; (9) The refreshment in the examination room should be curtailed to prevent removing of mask and increase the risk of exposure. Refreshments should only be served individually as packed foods/beverage in a dedicated area, maintaining adequate distance and proper hand hygiene. Eating in a group should be discontinued; (10) A backup plan for unforeseen events like electricity failure or poor Internet connectivity should always be ready to avoid undue interruption of the examination. Also, a standby technical support personnel should be available at all times.

The conduct of examination using virtual mode was entirely a new and enriching learning experience for us and it will go a long way for safe and smooth conduct of such examination in future.

\section{Conclusion}

Being for the first time and despite initial apprehensions and uncertainties, the examination process went off well to everyone's satisfaction, owing to the prior thoughtful planning and rigorous virtual trials. Though inability to examine and interact with the patient was a major limitation, in the present times, where safety of everyone is a priority, the virtual format is undoubtedly a safe and acceptable mode for conduct of examination quite similar to in-person evaluation.

Note

All the participants have given the consent for use of their images for publication in journal. The participants understand that their names and initials will not be published and due efforts will be made to conceal their identity, but anonymity cannot be guaranteed.

\section{Conflict of Interest}

None declared.

\section{References}

1 Ferrel MN, Ryan JJ. The impact of COVID-19 on medical education. Cureus 2020;12(3):e7492

2 Ahmed H, Allaf M, Elghazaly H. COVID-19 and medical education. Lancet Infect Dis 2020;20(7):777-778

3 Rose S. Medical student education in the time of COVID-19. JAMA 2020;323(21):2131-2132

4 Mossa-Basha M, Meltzer CC, Kim DC, Tuite MJ, Kolli KP, Tan BS. Radiology department preparedness for COVID-19: Radiology Scientific Expert Review Panel. Radiology 2020;296(2):E106-E112

5 Rajan R, Radhakrishnan DM, Srivastava AK, et al. Conduct of virtual neurology DM final examination during COVID-19 pandemic. Ann Indian Acad Neurol 2020;23(4):429-432 